\title{
Findings of an experimental study in a rabbit model on posterior capsule opacification after implantation of hydrophobic acrylic and hydrophilic acrylic intraocular lenses
}

\author{
Nikolaos Trakos' \\ Elli loachim² \\ Elena Tsanou² \\ Miltiadis Aspiotis' \\ Konstantinos Psilas' \\ Chris Kalogeropoulos' \\ 'University Eye Clinic of loannina, \\ loannina, Greece; ${ }^{2}$ Pathology \\ Department, University of Ioannina, \\ loannina, Greece
}

\begin{abstract}
Purpose: Study on cell growth on the posterior capsule after implantation of hydrophobic acrylic (Acrysof SA 60 AT) and hydrophilic acrylic (Akreos Disc) intraocular lenses (IOL) in a rabbit model and comparison of posterior capsule opacification (PCO).

Methods: Phacoemulsification was performed in 22 rabbit eyes, and two different IOL types (Acrysof SA60 AT and Akreos Disc) were implanted. These IOLs had the same optic geometry (square edged) but different material and design. Central PCO (CPCO), peripheral PCO (PPCO), Sommering's ring (SR) formation, type of growth, extension of PCO, cell type, inhibition, and fibrosis were evaluated three weeks after surgery. Histological sections of each globe were prepared to document the evaluation of PCO.

Results: No statistically significant difference was observed between a hydrophobic acrylic IOL and a hydrophilic acrylic IOL in relation to the CPCO, PPCO, type of growth, extension, cell type, inhibition, and fibrosis. Statistically significant difference was observed in relation to the formation of SR with Acrysof SA 60 AT group presenting more SR than Akreos Disc group. Conclusion: PCO was not influenced by the material of the IOL or the design of the haptics of the IOLs we studied.
\end{abstract}

Keywords: posterior capsule opacification, intraocular lenses, rabbit model

\section{Introduction}

Secondary cataract or posterior capsule opacification (PCO) is a result of cataract surgery; it is the most frequent long term complication in cataract surgery with an incidence of up to $50 \%$ according to some investigators (Apple et al 1992). This opacification may be created when cells are developed between posterior capsule and intraocular lenses (IOL) (Apple et al 1992). PCO reduces the visual acuity in the postoperative period and can be treated with YAG Laser, which acts at the posterior capsule by opening it and clearing the visual axis (Apple et al 1992). A dramatic decrease in PCO has been observed after the improvement of surgical techniques, which contributed to diminish the intraoperative and postoperative inflammation (Apple et al 2000). In some of the studies pharmaceutical substances like anti-mitotic agents, such as colchicine (Power et al 1994) and daunomycin (McDonnel et al 1988), have been used for the inhibition of cells' proliferation. The studies of PCO in a rabbit model offered a better understanding of the factors that assist the inhibition of PCO. The modification of the posterior edges of IOLs' optics from round to sharp, by creating a discontinuous bend, was critical in order to prevent the cells' growth to the posterior capsule, as Nishi and other researchers have already proved (Nishi et al 1998; Nishi and Nishi 1999). 
In the past it has been stated that cases with hydrophobic acrylic IOLs present the least PCO due to the increased adhesion of this material to the posterior capsule (Linnola et al 1999, 2000). In a collective study, Heatley and colleagues compared PCO in acrylic hydrophobic and hydrophilic IOLs and found that there was much less with the hydrophobic ones (Heatley et al 2005).

Only few reports have studied the influence of haptic design in PCO (Nishi et al 2004; Sacu et al 2005). There is increased evidence that there is migration of lens epithelial cells through haptic root of single-piece hydrophobic acrylic IOL (Acrysof) (Sugita et al 2004); although it is not clear how this affects the performance of this IOL in terms of PCO.

Although the edge design is more important than the material composition in hydrophobic acrylic and silicone IOLs, this has not been shown in various designs and types of hydrophilic acrylic materials (Findl et al 2005).

In a clinical study, the PCO rate with the Akreos IOL was similar to that with other acrylic IOLs reported in the literature (Khandwara et al 2007). For all the above reasons we decided to perform a clinicopathological study in a rabbit model in order to evaluate PCO by comparing two different types of IOLs, which would have a square optic edge but different material compositions (hydrophobic acrylic and hydrophilic acrylic). To the best of our knowledge no comparative study of these IOLs has been performed in the past.

We aimed to detect whether we can have low and comparable PCO in these two IOLs without using any of the inhibitory pharmaceutical substances. We also aimed to study the cell type, type of growth, and extension of PCO in correlation to each of these two different IOLs. Finally, we aimed to extrapolate useful findings from the pathological view of this experimental study that might help to the investigation of the factors that inhibit PCO.

\section{Materials and methods}

Twenty two Dutch Belted pigmented serum Pasterella-free rabbits were housed and cared in the Animal Breading Unit of the University of Ioannina, Greece. They were of the same age ( 10 weeks) and weight $(2.5 \mathrm{~kg})$. They were treated according to the ARVO statement.

The types of IOLs randomly implanted were:

1) Acrysof SA60AT (Alcon Laboratories Inc., Fort Worth, TX, USA) 1-piece, hydrophobic acrylic IOLs with hydrophobic haptics and $0^{\circ}$ angulation.

2) Akreos Disc (Bausch and Lomb, Athens, Greece) 1-piece, hydrophylic acrylic IOLs with two hydrophylic fenestrated plate haptics and $0^{\circ}$ angulation.
These foldable IOLs had the same optic diameter $(6 \mathrm{~mm})$ with a slightly different total diameter (Acrysof SA60AT has $13 \mathrm{~mm}$ and Akreos Disc has $10.7 \mathrm{~mm}$ ). The haptics angulation was $0^{\circ}$. A study of the edges of the optic and haptics of these IOLs was performed with the scanning electron microscope (SEM) at the University of Ioannina. The IOLs used had a sharp posterior optic edge (Figure 1).

Although these IOLs had posterior square-edged optic, they had different material composition of the optic and haptics and also different haptics design and optic-haptic junction. We examined each of the different features and how this influenced PCO, and also examined the extension of PCO, cell type, and type of growth on the posterior capsule. We compared them for this purpose. All operations were performed by the same surgeon (NT).

Each rabbit was anesthetized with an intramuscular injection of ketamine hydrochloride $(50 \mathrm{mg} / \mathrm{Kg})$ and xylazine $(7 \mathrm{mg} / \mathrm{kg}$ ) and prepared for surgery by pupil dilation. Phacoemulsification was performed with a Phaco-Plus (Advanced Medical Optics, Santa Ana, CA, USA) using an aseptic technique and a Möller-Wedel surgical microscope. A $3.2 \mathrm{~mm}$ clear corneal incision was performed and a viscoelastic Healon-5 (Pharmacia, Uppsala, Sweden) was used to facilitate a 5-5.5 mm continuous circumferential capsulorrhexis (CCC) overlapping the optic. After hydrodissection and phacoemulsification, removal of the lens material was performed without leaving any residual cortical fibres. The pupil dilation and reduction of intraoperative inflammation was maintained during surgery by using $0.5 \mathrm{~mL}$ of epinephrine 1:1000 and $0.5 \mathrm{~mL}$ heparin $(10,000 \mathrm{USP}$ units $/ \mathrm{mL})$ added to each $500 \mathrm{~mL}$ of irrigation solution. The capsular bag was inflated with viscoelastic material and a +21 diopter IOL was implanted in the bag. Thorough aspiration of the viscoelastic was performed to ensure complete removal of the viscoelastic. No suture was used to close the wound. A mixture of $0.25 \mathrm{cc}$ dexamethasone and $0.25 \mathrm{cc}$ gentamycin was injected subconjunctivaly at the end of the surgery. All procedures were documented using a Sony DVD recorder.

Postoperatively, the eyes were treated with cyclopentolate drops and a combination of dexamethasone and neomycin ointment twice a day for three weeks in order to suppress inflammatory factors. All rabbits were evaluated at day 1 and at 1,2, and 3 weeks for ocular inflammatory response, corneal edema and $\mathrm{PCO}$, and retroillumination photos were taken with the pupil dilated. Euthanasia was performed with a $2.0 \mathrm{cc}$ intravenous injection of sodium pentobarbital.

Afterwards enucleation of the globes was performed. Neutral buffered formalin $10 \%$ solution for 24 hours was 


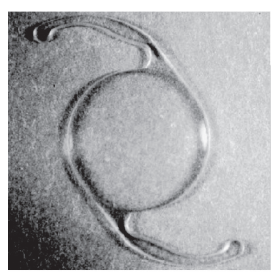

A

Overview

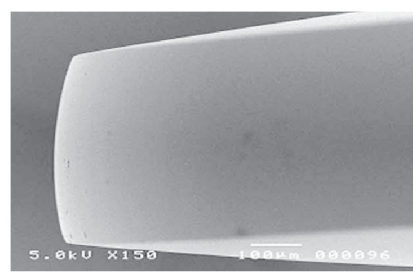

B

SEM photo of the optic

AcrySof SA60AT (Alcon)

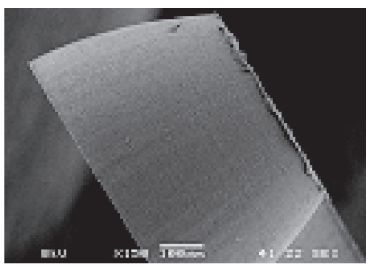

C

SEM photo of the haptic

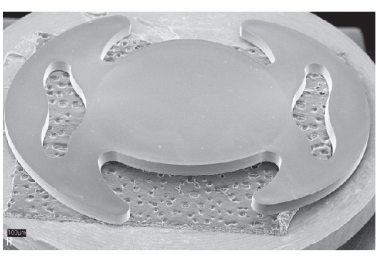

D

Overview

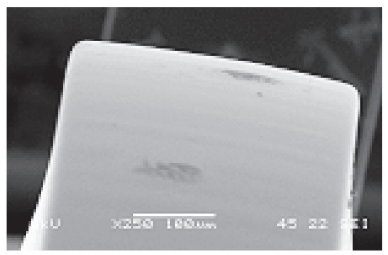

SEM photo of the optic

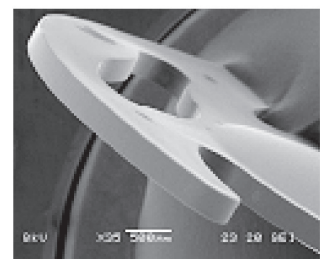

F

SEM photo of the haptic

Akreos Disc (Bausch \& Lomb)

Figure I In the photos above you note the sharp posterior edge of the IOLs used in the study. A) Overview of AcrySof SA60AT (Alcon). B) Scanning electron microscopy (SEM) photo of the optic of AcrySof SA60AT showing it's sharp posterior edge. C) SEM photo of the haptic of AcrySof SA60AT, showing that it has the same sharp edge as the optic. D) Overview of Akreos Disc (Bausch and Lomb). E) SEM photo of the sharp posterior edge of the optic of Akreos Disc. F) SEM photo of the haptic of Akreos Disc showing that it has sharp egde as well.

Note: Courtesy of Alcon and Bausch and Lomb and the Department of SEM, University of loannina.

used to fixate the globes. The globes were then bisected coronally just anterior to the equator. Gross examination and photographs from the posterior aspect (Miyake-Apple posterior view) were performed to evaluate PCO.

Central PCO was evaluated in a central part of 3-mm diameter of the IOL optic corresponding to the pupillary area (light grey area in the scheme), and peripheral PCO was evaluated in the peripheral part corresponding to the area including the IOL optic outside the $3.0 \mathrm{~mm}$ pupillary area (dark grey area). Sommering's ring (SR), which is a white ring of cells in the periphery of the bag, was evaluated in the area around the optic (white area) (Figure 2).

The intensity of central PCO, peripheral PCO, and SR were scored according to the methods established in previous experimental studies (Figure 3) (Nishi et al 2000).

The intensity of PCO was graded from 0 to 3 as follows: none (0) was characterized as absence of cells, slight $(+1)$ as presence of few cells and when the iris pattern was still detectable, obvious $(+2)$ as significant presence of cells or focally covered areas and when the iris pattern was barely detectable, and distinct $(+3)$ when a lot of cells were present and the iris pattern was not detectable in certain areas. SR was scored from $0-4$. For SR we applied the same criteria (Nishi et al 2000), but in addition grade 4 was characterized by the appearance of intense white material (see example of SR grade 4 in Figure 7).

We used the classical evaluation system for the Soemmering's ring: we divided the area in four quadrants and then estimated the SR intensity (SRI)/SR area (SRA) in each of the four quadrants providing the mean SRI (MSRI) with the formula SRI/SRA $=[(\mathrm{SRI} 1)+(\mathrm{SRI})+(\mathrm{SRI} 3)+$ (SRI4)]/4 (Figure 4).

We noted if the CCC was on the optic, if the IOL was in or out of the bag and if there was distortion of the haptics. We ruled out cases with CCC out of the optic/bag, posterior capsule tear, intense inflammation, endophthalmitis, or when the follow-up was less than three weeks.

\section{Histologic study}

All globes were sectioned in a pre-equatorial level and the anterior segments stained with hematoxylin and eosin, periodic acid-Schiff and Masson's trichrome. The specimens 


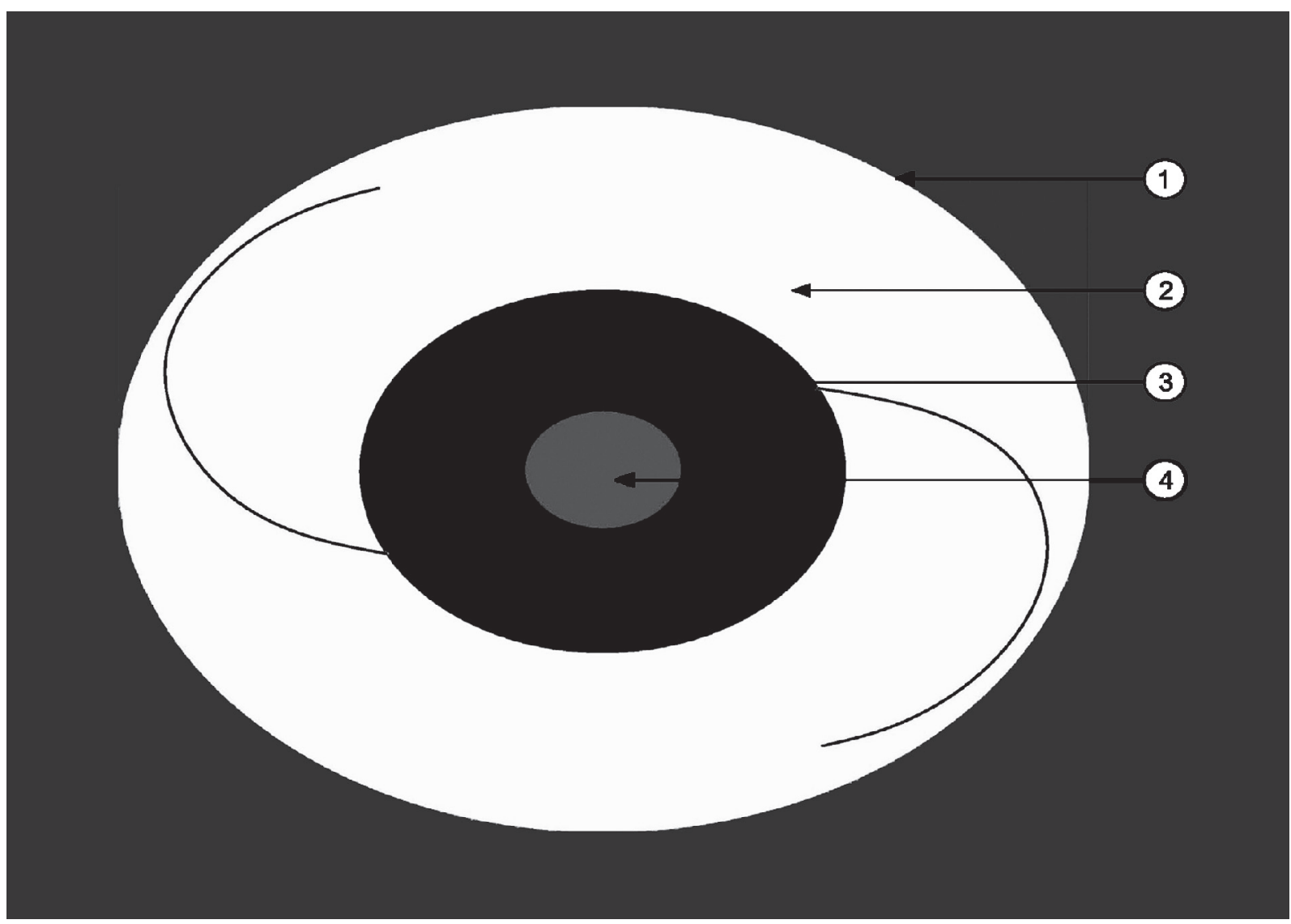

Figure 2 Schematic illustration of the IOL (optic with the haptics) in the capsular bag (arrow I = lens equator). Note the area around the optic (arrow 2, white area), where Sommering's ring was evaluated, the peripheral area of the optic (arrow 3, dark grey), where peripheral PCO was evaluated and the central area of the optic (arrow 4, light grey area), where central PCO was evaluated.

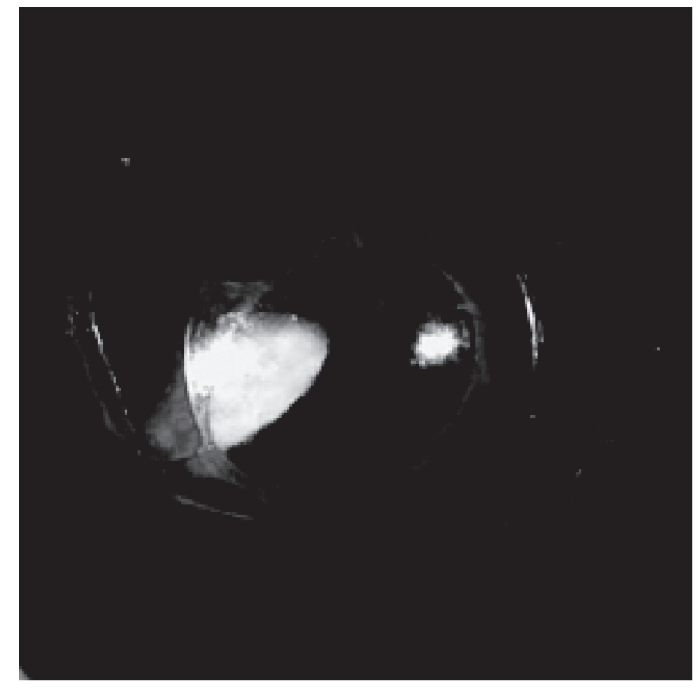

A

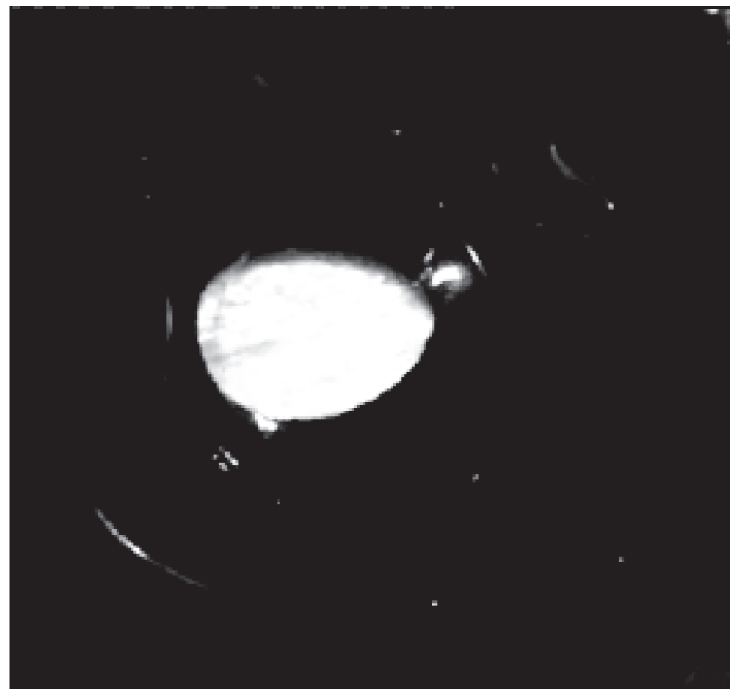

B

Acrysof SA 60 AT

Akreos Disc

Figure 3 Gross photographs from behind in the rabbit model we studied (Miyake-Apple technique). A) Note the PCO developed in a case with Acrysof SA 60 AT. You can also note the fibrous strands behind the optic. B) Note the mild PCO developed in a case with Akreos Disc.

Abbreviation: PCO, Posterior capsule opacification. 


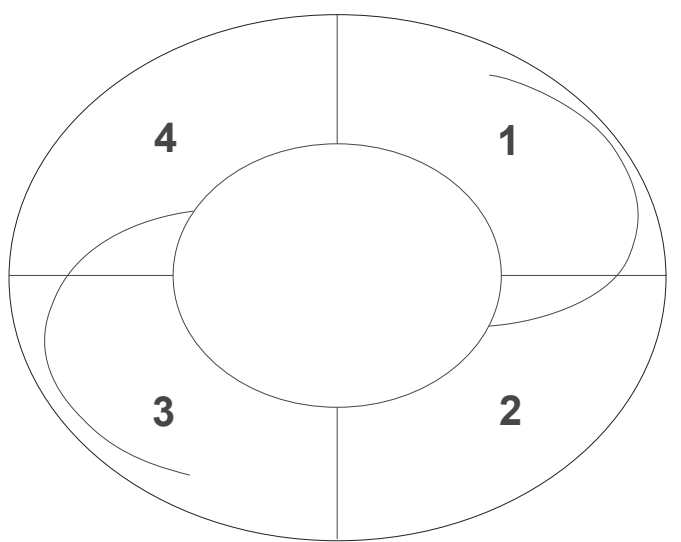

Figure 4 Note the area around the optic divided in four different quadrants $(1,2,3,4)$ each of them evaluated separately according to the intensity of Sommering's ring $(\mathrm{SRI})$. The mean SRI was calculated by the formula MSRI $=[(\mathrm{SRII})+(\mathrm{SRI})+(\mathrm{SRI})+$ $(\mathrm{SRI})] / 4$.

were processed in a way to ensure that the optic-haptic junctions would be included in the histopathologic cuts (Figure 5).

We decided to evaluate the extension of cells' growth on the posterior capsule (none, $25 \%, 25 \%-50 \%, 50 \%-75 \%$, $75 \%-100 \%, 100 \%$ ), the type of growth (continuous/ discontinuous) and the cell type pattern (balloon cells, myofibroblast cells).

Immunohistochemistry was performed. Epithelioform cells (anterior lens epithelial cells, equatorial cells and bladder cells) were stained with pancytocin. Myofibroblasts were stained with vimentin. The identification of these cells was also assisted by their special morphological features in microscope. The presence of fibrosis (identified with the Masson's trichrome stain as none, little and intense) was also recorded. Sections without enough posterior capsule were ruled out.

In previous studies, it has not been defined exactly what inhibition was. In some of these studies inhibition was considered when a posterior capsule was totally or relatively free of cells or having a single layer of cells (Linnola et al 2000; Nishi et al 2001). We defined as inhibition of cells' growth when the extension of cells would be less than $25 \%$.

\section{Statistical analysis}

To determine whether there was a difference between the two groups in central PCO (CPCO), peripheral PCO (PPCO) and MSRI statistical analysis was performed with the SPSS software programme version 11.5 using the nonparametric Mann-Whitney test for one-way ANOVA.

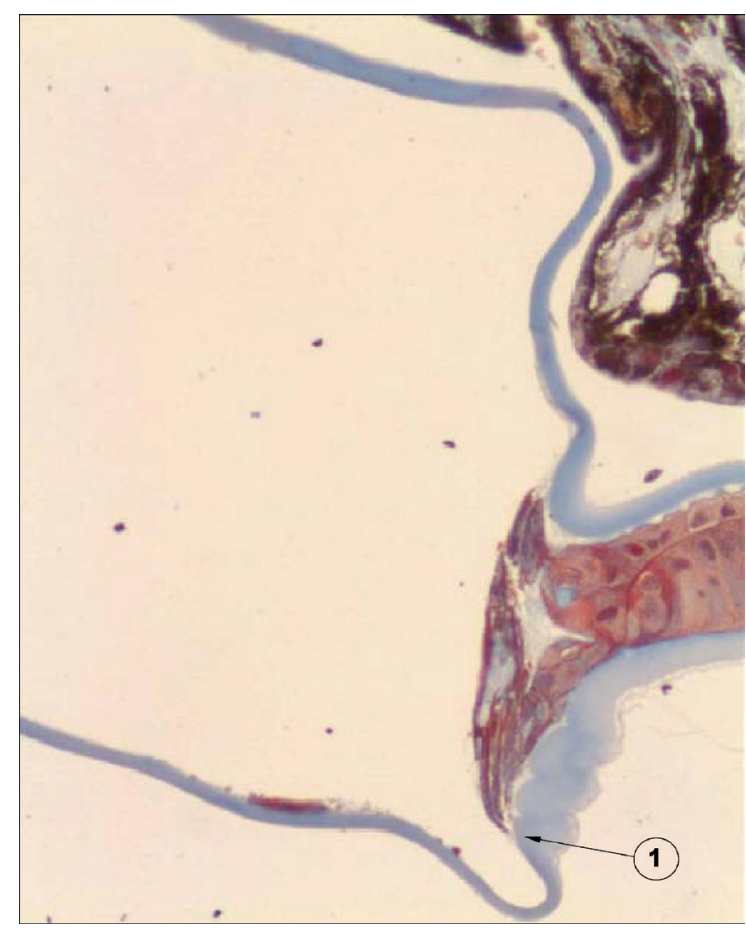

Figure 5 Photomicrograph of the anterior segment of an eye of the rabbit model. Note the effective barrier created by the sharp posterior edge of an Acrysof SA60 AT, reflecting the inhibition of cells' growth (arrow). Note the thicker anterior capsule and the thinner posterior capsule. The IOL optic and haptics are dissolved out of the section, leaving a large space between anterior and posterior capsule. Note: Masson's trichrome stain; original magnification $\times 200$.

\section{Results}

In the follow-up period, no endophthalmitis was noticed and none of the eyes were ruled out. Mild corneal edema at the site of the incision was observed in most of the cases. Mild inflammatory reaction was noted during the first week.

Twenty two eyes were studied at the end of the three weeks follow-up. We compared 10 Acrysof SA60AT (Alcon Laboratories Inc.) to 12 Akreos Disc (Bausch and Lomb). The scores of CPCO, PPCO, and MSRI are given in a histogram (Figure 6).

No statistically significant difference in $\mathrm{CPCO}(\mathrm{P}=0.539)$ or in PPCO $(\mathrm{P}=0.093)$ was noted between the two types of IOLs. The CPCO was low in both groups.

However, there was statistically significant difference in SR using the MSRI scoring system $(\mathrm{P}=0.004)$. In particular there was difference between Acrysof SA60AT and Akreos Disc with both the Mann-Whitney test and the Spearman's test.

Distortion of haptics combined with more intense SR was noticed in two of the Acrysof SA60AT IOLs (Figure 7).

In one of the specimens of the Acrysof SA60AT proliferation of the cells was noticed on the optic-haptic junction supporting the theory that this junction maybe the "weak" 


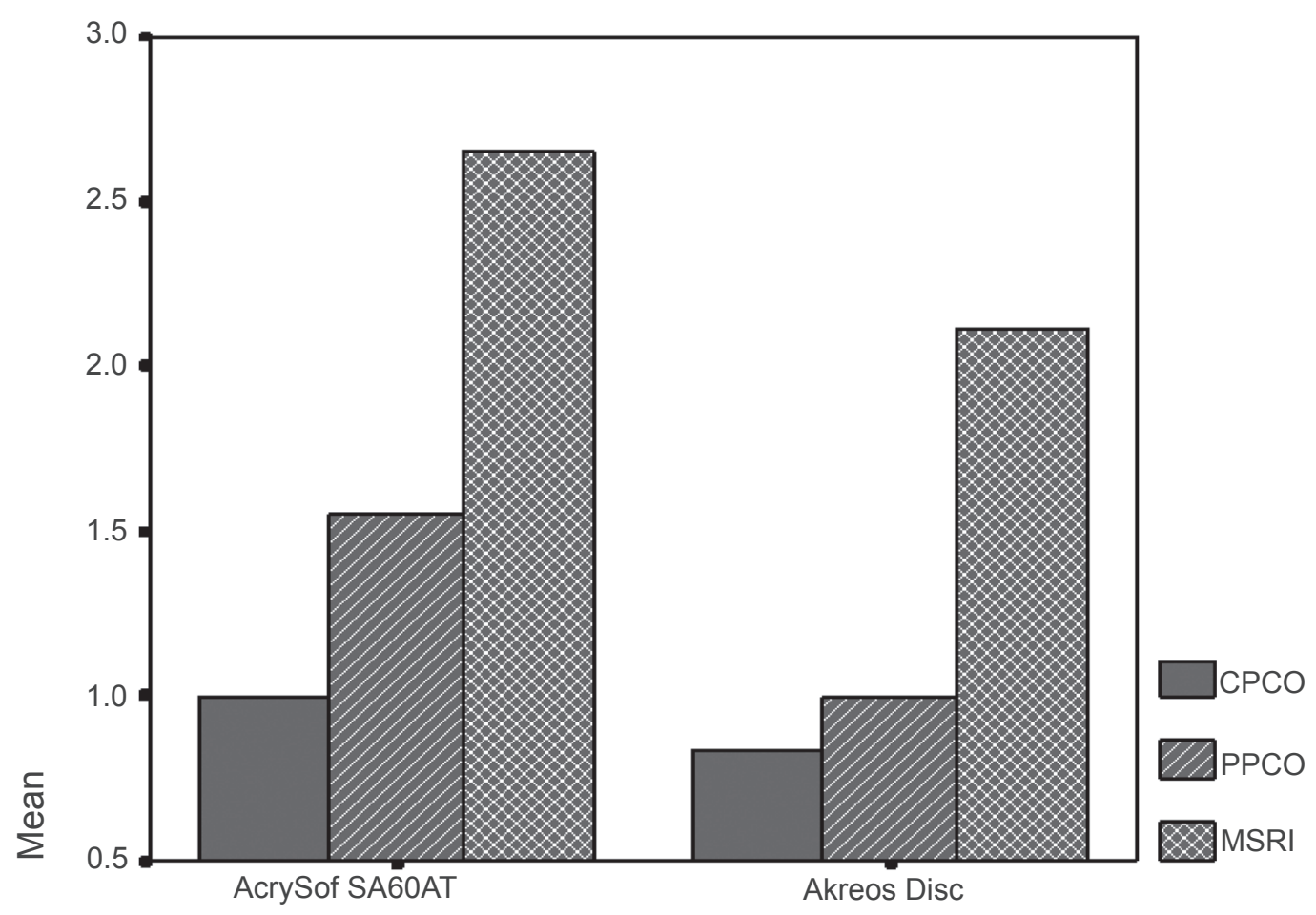

Type IOL

Figure 6 In this histogram the median scores of central posterior capsule opaciification (CPCO), peripheral PCO (PPCO) and median Sommering's ring intensity (MSRI) of the two groups of intraocular lenses (IOLs) are depicted in axis Y. Note the statistically significant more MSRI scores in Acrysof SA 60 AT group (see photograph of intense Sommering's ring of this IOL group in Figure 7). The scores of CPCO and PPCO in Acrysof SA 60 AT group are not statistically significant although they are higher than the ones of Akreos Disc group.

point for the extension of cells on the posterior capsule (Figure 8).

In order to study the extension of cells in the posterior capsule we needed to have specimens with a long enough posterior capsule. However a significant number of the specimens were short and we ruled them out regarding the study of the extension. Nevertheless the number of specimens we studied was enough to extrapolate useful results statistically significant.

We correlated the pattern of cells' growth, the grade of extension, inhibition, fibrosis, and the cell type with the number of each IOL type (Table 1).

There was no statistically significant difference between hydrophilic and hydrophobic IOL types when we correlated them with fibrosis $(P=0.97)$, extension $(P=0.22)$, growth type $(\mathrm{P}=0.62)$, cell type $(\mathrm{P}=0.1)$, and inhibition of cell growth into the posterior capsule $(\mathrm{P}=0.91)$. Finally, there was no statistically significant correlation between cell type and fibrosis $(\mathrm{P}=0.59)$.

\section{Discussion}

In our experimental study we did not find any statistically significant difference in comparing PCO in both IOLs. The only known differences between them were the material and the haptics design. From our study it seems that the inhibitory effect is similar regardless of whether the acrylic material of IOL is hydrophilic or hydrophobic.

The main factor for the inhibition of the cells' growth is the creation of a mechanical obstacle in the posterior capsule by the sharp edge of the IOL. The sharp edges in combination with the creation of a capsular bend contribute to this inhibition (Nishi and Nishi 1999). Studies by Nishi and colleagues conducted to the conclusion that the material is less important than the optic geometry in prevention of PCO (Nishi et al 1998; Nishi and Nishi 1999). Linnola and colleagues (2000) proved that hydrophobic acrylic material presents higher adhesion to the posterior capsule in comparison to other materials.

Ursell and colleagues (1998) in a collective study reported that the design of the IOL is important for the inhibition of PCO, but the material of the IOL and the quality of the IOL's surface are also important for this inhibition. Various authors have described higher frequency of PCO in IOLs made of poly (methyl methacrylate) or hydrogel (Ursell et al 1998; Nishi et al 2002). The capsule is adhered around 


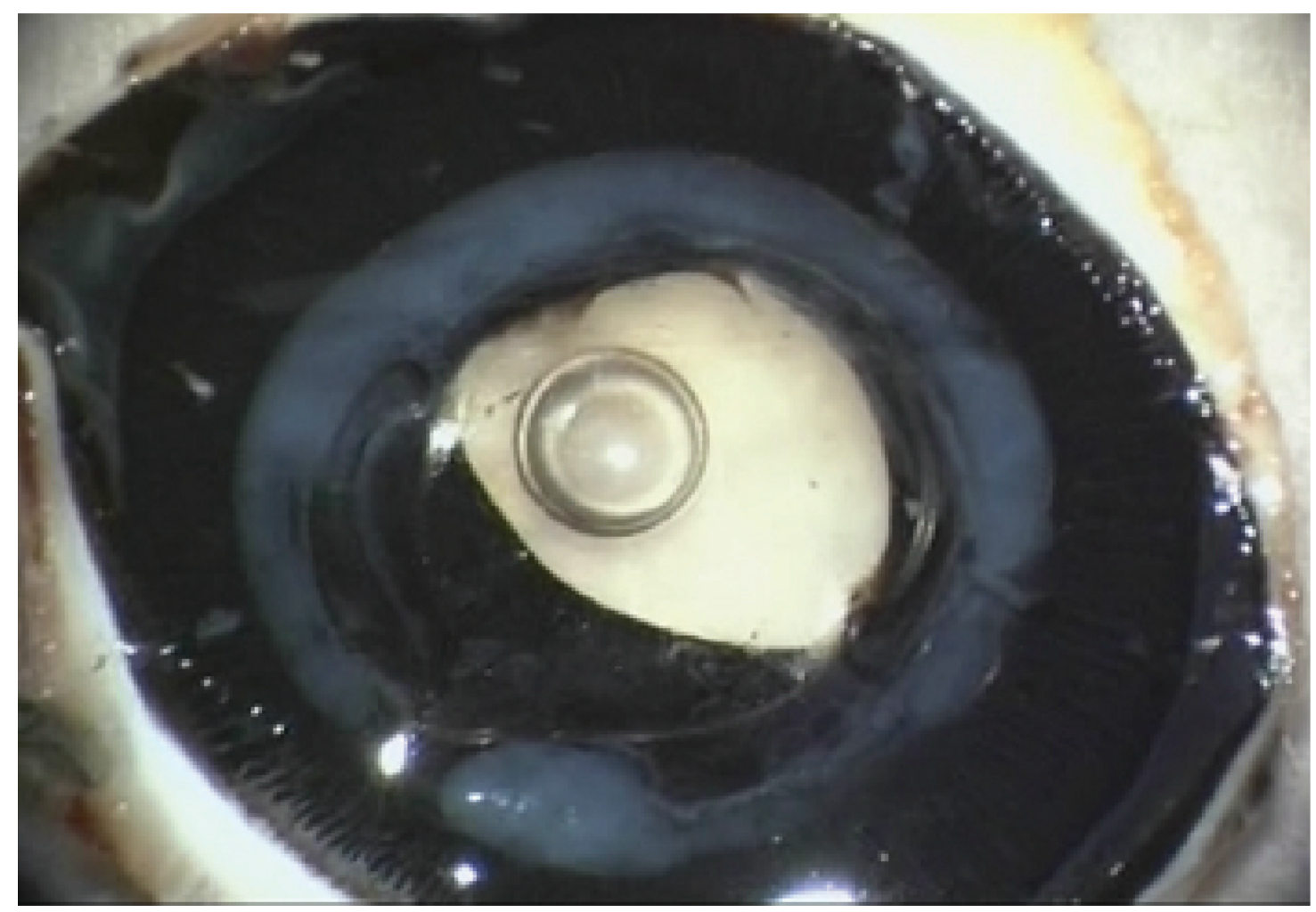

Figure 7 Gross photograph (Miyake-Apple posterior view technique) showing an Acrysof SA 60 AT. You can see the intense Sommering's ring (grade 4, white ring). Note the distortion of the haptics of the intraocular lens which are compressed by Sommering's ring and are bent over the optic.

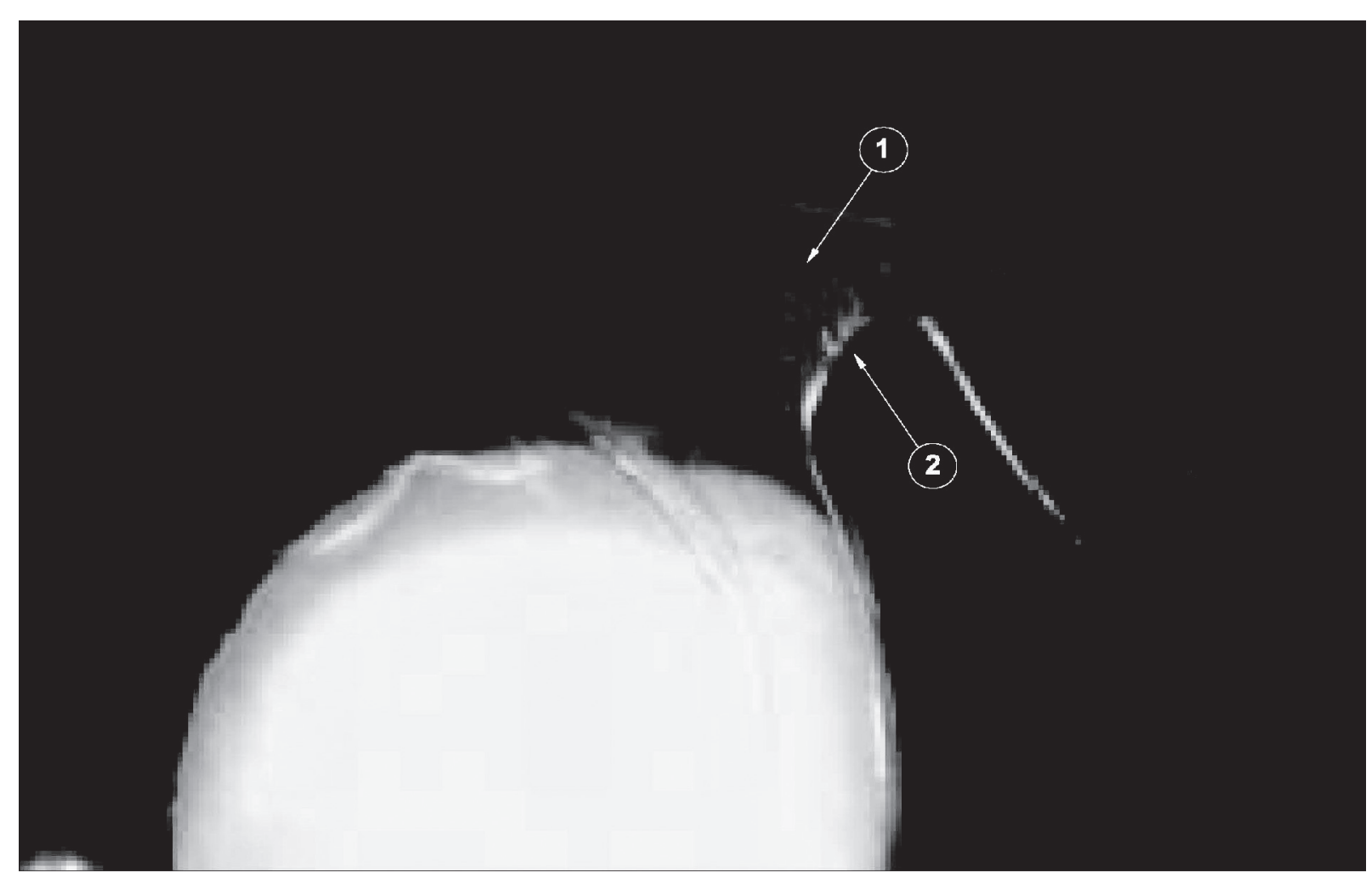

Figure 8 Photo showing lens epithelial cells starting their migration, from the periphery to the center, through the optic-haptic junction of I-piece Acrysof SA 60 AT (whitish area on the junction). Arrow I shows the route of cells on the optic-haptic junction and arrow 2 shows the area where these cells seem to be directed, contributing to posterior capsule opaciification formation. 
Table I Correlations of growth, cell type, extension, inhibition and fibrosis and numbers of each type of intraocular lenses (IOL); note that none of the AcrySof IOLs presented intense fibrosis on the posterior capsule, and two Akreos Disc presented intense fibrosis

\begin{tabular}{|c|c|c|c|}
\hline & \multicolumn{3}{|l|}{ IOL types } \\
\hline & AcrySof SA 60 AT & Akreos disc & $P$ value \\
\hline Growth & Number of IOLs & Number of IOLs & \\
\hline Continuous & I & 2 & $P=0.628$ \\
\hline Discontinuous & 6 & 4 & \\
\hline \multicolumn{4}{|l|}{ Cell type } \\
\hline Balloon cells & 2 & 2 & \\
\hline Myofibroblasts & 3 & 2 & $P=0.100$ \\
\hline Mixed & 2 & 2 & \\
\hline \multicolumn{4}{|l|}{ Extension } \\
\hline $0 \%-25 \%$ & 3 & 3 & \\
\hline $25 \%-50 \%$ & 4 & 1 & \\
\hline $50 \%-75 \%$ & I & 0 & $P=0.220$ \\
\hline $75 \%-100 \%$ & I & 2 & \\
\hline $100 \%$ & I & 0 & \\
\hline \multicolumn{4}{|l|}{ Inhibition } \\
\hline$<25 \%$ & 7 & 8 & $P=0.918$ \\
\hline$>25 \%$ & 3 & 3 & \\
\hline \multicolumn{4}{|l|}{ Fibrosis } \\
\hline None & 8 & 9 & \\
\hline Little & 2 & 0 & $P=0.973$ \\
\hline Intense & 0 & 2 & \\
\hline
\end{tabular}

the optic rim, taking a certain configuration, the so-called capsular bend configuration, which is important for the inhibition of PCO. Sacu and colleagues (2005) observed that PCO was less in eyes where the posterior capsule was taught around the optic rim with a type $\mathrm{W}$ configuration. This type was more frequently noticed in silicone IOLs (Sacu et al 2005).

All these studies show contradictory results. Certainly a lot of factors contribute to the inhibition of PCO, but the conclusion from our study is that it seems that, regardless whether the hydrophobic or the hydrophilic nature of the acrylic material, the rate of PCO is similar.

Nishi's theory is that capsular adhesion is a prerequisite of capsular bend formation (Nishi and Nishi 1999). A sharp optic edge alone does not provide a substantial barrier when a capsular bend is not formed. In our experimental study a statistically significant increase in SR intensity was found at 1-piece Acrysof.

Werner and colleagues (2005) did not find any statistically significant difference in SR intensity comparing 1-piece
Acrysof to 3-piece Acrysof. It has been proposed that there might be a discontinuous capsular bend or a not well formed rectangle in 1-piece Acrysof as the bulky haptics are the cause (Werner et al 2005). It has also been observed that this kind of haptics may be distorted in few cases (Vargas et al 2002).

A bulky haptic, such as the haptics of foldable singlepiece IOLs, and a large optic may hamper capsular adhesion and bend formation. Akreos Disc also has a bulky haptic and a large optic. The difference in SR between Acrysof and Akreos may be explained by the fact that the haptics of the Acrysof SA60AT were found to be distorted in $20 \%$ of cases in our study by not being able to inhibit the aggregation of the cells in the equatorial level of the capsule. This does not happen with the haptics of Akreos that are compact.

The type of adhesion at the level of the optic may play an important role and also the speed of capsular bend formation might be variable (Werner et al 2002); these factors influence the development of cells in the posterior capsule. Bulky haptics of 1-piece Acrysof may hamper the adhesion between anterior and posterior capsule (Werner et al 2002).

The theory that hydrophobic acrylic IOLs present less PCO was based to the fact that the material of these IOLs is connected better to the posterior capsule as mentioned above. These IOLs are connected better with fibronectin. However, the hydrophilic acrylic IOLs are connected better with laminin and collagen type IV which are also important proteins of the extracellular matrix (Linnola et al 1999). Even if the role of fibronectin would be more important than the other proteins, factors previously mentioned such as speed of capsule adhesion and more fibrosis (in IOLs made of hydrophilic acrylic material) which tends to seal the anterior and posterior capsule would decompensate this effect (Linnola et al 2000; Nishi et al 2002). Furthermore we must mention that in our study we confirmed the observation that the optic-haptic junctions of square-edged 1-piece Acrysof IOLs may represent a site for cell ingrowth and PCO formation, being the Achile's heel for this kind of IOL. At the level of these junctions, the barrier effect of the square edge appears to be less effective (Findl et al 2005; Werner et al 2005). The difference in the results of various studies might also be explained by the regression of the epithelial cells of PCO which has been noticed (Boehl et al 2005).

Another important observation is that the obstacle created by the adhesion of the anterior with the posterior capsule, cannot be formed and will not have appropriate efficiency, if fibrosis will not maintain adhered the anterior with the posterior capsule. For this reason fibrosis of a certain small 
grade is desirable and it contributes to the reduction of PCO. IOLs which have a higher potential of myofibroblasts development, such as hydrophilic acrylic are favored in comparison to IOLs with less fibrogenesis potential. For this reason hydrophilic acrylic decompensate the referred disadvantage of less adhesion with fibronectin and they prevent PCO in a similar grade.

It remains to be shown if this is applied to the silicone IOLs as well. In a pilot clinical study evaluation of posterior capsule opacification after implantation of the Akreos Disc and Akreos Fit acrylic intraocular lenses showed low PCO rate (Späth et al 2003).

In our experimental study in a rabbit model we evaluated PCO comparing Akreos Disc to Acrysof SA60AT. We need though to investigate what happens in comparison to the silicone IOLs which requires further studies with more IOLs. We also need to extend the study in other types and designs of IOLs. In this study we examined what happens in the area between the posterior capsule and the optic, but further studies are needed to examine what happens to the side of the optic and the area of adhesion of the anterior and posterior capsule as well.

\section{Conclusion}

Posterior capsule opaciification may be reduced to a clinically negligible level by using surgical techniques that enable us to perform cataract surgery with minimal inflammation and by optimizing the design and the material of an IOL. PCO is not influenced by the material of the intraocular lens or the design of the haptics of the IOLs we studied.

Hydrophilic acrylic IOLs seem to have similar potential to hydrophobic acrylic IOLs in inhibiting cell growth on the posterior capsule. Further studies are needed to see if this is applied for other types of IOLs made of hydrophilic acrylic material and silicone IOLs.

\section{Disclosure}

The authors have no proprietary or conflict of interest in this work.

\section{References}

Apple DJ, Peng Q, Visessook N, et al. 2000. Surgical prevention of posterior capsule opacification. Part 1: Progress in eliminating this complication of cataract surgery. J Cataract Refract Surg, 26:180-7.

Apple DJ, Solomon KD, Tetz MR, et al. 1992. Posterior capsule opacification. Surv Ophthalmol, 37:73-116.

Buehl W, Findl O, Neumayer T, Georgopoulos M, Sacu S. 2005. Short-term changes in the morphology of posterior capsule opacification. J Cataract Refract Surg, 31:962-8.
Findl O, Menapace R, Sacu S, Buehl W, Rainer G. 2005. Effect of optic material on posterior capsule opacification in intraocular lenses with sharp-edge optics. Randomized clinical trial. Ophthalmology, 112:67-72.

Heatley CJ, Spalton DJ, Kumar A, Jose R, Boyce J, Bender LE. 2005. Comparison of posterior capsule opacification rates between hydrophilic and hydrophobic single-piece acrylic intraocular lenses. J Cataract Refract Surg, 31:718-24.

Khandwala MA, Marjanovic B, Kotagiri AK, Teimory M. 2007. Rate of posterior capsule opacification in eyes with the Akreos intraocular lens. $J$ Cataract Refract Surg, 33:1409-13.

Linnola RJ, Sund M, Ylonen R, Pihlajaniemi T. 1999. Adhesion of soluble fibronectin, laminin, and collagen type IV to intraocular lens materials. $J$ Cataract Refract Surg, 25:1486-91.

Linnola RJ, Werner L, Pandey SK, Escobar-Gomez M, Znoiko SL, Apple DJ. 2000. Adhesion of fibronectin, vitronectin, laminin, and collagen type IV to intraocular lens materials in pseudophakic human autopsy eyes. Part 1: histological sections. J Cataract Refract Surg, 26:1792-806.

McDonnell PJ, Krause W, Glaser BM. 1988. In vitro inhibition of lens epithelial cell proliferation and migration. Ophthalmic Surg, 19:25-30.

Nishi O, Nishi K, Akura J, Nagata T. 2001. Effect of round-edged acrylic intraocular lenses on preventing posterior capsule opacification. $J$ Cataract Refract Surg, 27:608-13.

Nishi O, Nishi K, Akura J. 2002. Speed of capsular bend formation at the optic edge of acrylic, silicone, and poly-methyl methacrylate lenses. $J$ Cataract Refract Surg, 28:431-7.

Nishi O, Nishi K, Osakabe Y. 2004. Effect of intraocular lenses on preventing posterior capsule opacification: design versus material. J Cataract Refract Surg, 30:2170-6.

Nishi O, Nishi K, Sakanishi K. 1998. Inhibition of migration lens epithelial cells at the capsular bend created by the rectangular optic edge of a posterior chamber intraocular lens. Ophthalmic Surg Lasers, 29:587-94.

Nishi O, Nishi K, Wickstrom K. 2000. Preventing lens epithelial cell migration using intraocular lenses with sharp rectangular edges. J Cataract Refract Surg, 26:1543-9.

Nishi O, Nishi K. 1999. Preventing posterior capsule opacification by creating a discontinuous sharp bend in the capsule. $J$ Cataract Refract Surg, 25:521-6.

Power WJ, Neylan D, Collum LM. 1994. Daunomycin as an inhibitor of human lens epithelial cell proliferation in culture. J Cataract Refract Surg, 20:287-90.

Sacu S, Findl O, Menapace R, Buehl W. 2005. Influence of optic edge design, optic material, and haptic design on capsular bend configuration. J Cataract Refract Surg, 31:1888-94.

Späth U, Liekfeld A, Hartmann C, Pham DT. 2003. Evaluation of posterior capsule opacification after implantation of the Akreos Disc and Akreos Fit acrylic intraocular lenses - pilot studies. Klin Monatsbl Augenheilkd, 220:695-8.

Sugita M, Kato S, Sugita G, Oshika T. 2004. Migration of lens epithelial cells through haptic root of single-piece acrylic-foldable intraocular lens. Am J Ophthalmol, 377-9.

Ursell PG, Spalton DJ, Pande MV. 1998. Relationship between intraocular lens biomaterials and posterior capsule opacification. $J$ Cataract Refract Surg, 24:352-60.

Vargas LG, Peng Q, Apple DJ, et al. 2002. Evaluation of 3 modern single-piece foldable intraocular lenses: Clinicopathological study of posterior capsule opacification in a rabbit model. $J$ Cataract Refract Surg, 28:1241-50.

Werner L, Mamalis N, Izak A, et al. 2005. Posterior capsule opacification in rabbit eyes implanted with 1-piece and 3-piece hydrophobic acrylic intraocular lenses. J Cataract Refract Surg, 31:805-11. 